Nagumo, Mitio and

Isé, Kusuo

Osaka Math. J.

9 (1957), 221-234

\title{
On the Normal Forms of Differential Equations in the Neighborhood of an Equilibrium Point
}

\author{
By Mitio Nagumo and Kusuo Isé
}

\section{$\S 1$. Introduction.}

1. In this note we use the notations $\partial_{i} u$ and $\partial_{i j}^{2} u$ for $\frac{\partial}{\partial x_{i}} u$ and $\frac{\partial^{2}}{\partial x_{i} \partial x_{j}} u$ respectively. The vectors $\left(x_{1}, \cdots, x_{m}\right)$ and $\left(y_{1}, \cdots, y_{m}\right)$ in $R^{m}$ will be denoted briefly by $x$ and $y$ respectively.

Let $A=\left(a_{i j}\right)$ be a constant real $(m, m)$-matrix, all of whose characteristic roots $\lambda_{i}(i=1, \cdots, m)$ have non-zero real parts, and $f(x)=\left(f_{1}(x), \cdots, f_{m}(x)\right)$ a real vector function of class $C^{1}$ on some neighborhood of $x=0$, such that $f(0)=0$ and $\left|\partial_{x} f(x)\right| \leqq K \cdot|x|$ with a constant $K>0$ where

$$
|x|=\left(\sum_{i} x_{i}^{2}\right)^{\frac{1}{2}}, \quad\left|\partial_{x} f(x)\right|=\left\{\sum_{i, j}\left(\partial_{i} f_{j}(x)\right)^{2}\right\}^{\frac{1}{2}} .
$$

We consider the autonomous systems

$$
\frac{d x}{d t}=A \cdot x+f(x)
$$

and

$$
\frac{d y}{d t}=A \cdot y
$$

regarding $x, y$ and $f(x)$ as the column-vectors. The purpose of this note is to show that, under some conditions on $\lambda_{i}(i=1, \cdots, m)$ and $f(x)$, the system (1.1) can be transformed into (1.2) by a change of variables

$$
y=x+u(x)
$$

where $u(x)=\left(u_{1}(x), \cdots, u_{m}(x)\right)$ is a real vector function of class $C^{1}$, such that

$$
\left\{\begin{array}{l}
u(0)=0 \\
\left|\partial_{x} u(x)\right| \leqq L \cdot|x|
\end{array}\right.
$$

with some constant $L>0$. 
When $f(x)$ is analytic regular in $x$, in order to show the existence of the transformation given by (1.3) with analytic regular $u(x)$, we must necessarily assume that there exist no relations of the form

$$
\lambda_{i}=\sum_{j=1}^{m} n_{j} \cdot \lambda_{j}
$$

where $n_{j}(j=1, \cdots, m)$ are non-negative integers such that $\sum_{j=1}^{m} n_{j}>1$. As to this case, some results were obtained by H. Poincaré, C. L. Siegel, and others, while we obtain the present result for the real systems with a transformation of class $C^{1}$ under some weaker conditions.

\section{§ 2. Main Theorem.}

2. Theorem. Assumptions:

(i) A is a constant real $(m, m)$-matrix, all of whose characteristic roots $\lambda_{i}(i=1, \cdots, m)$ have non-zero real parts: $\Re\left(\lambda_{i}\right) \neq 0(i=1, \cdots, m)$. (ii) Let

$$
f_{i}(x)=p_{i}(x)+q_{i}(x) \quad(i=1, \cdots, m)
$$

where $p_{i}(x)$ are polynomials in $x$ with real coefficients such that $p_{i}(0)=\partial_{j} h_{i}(0)=0(i=1, \cdots, m ; j=1, \cdots, m)$, and $q_{i}(x)(i=1, \cdots, m)$ are real-valued functions of class $C^{1}$ satisfying

$$
\left\{\begin{array}{l}
q(0)=0 \\
\left|\partial_{x} q(x)\right| \leqq Q \cdot|x|^{h}
\end{array}\right.
$$

with some integer $h>0$ and some constant $Q>0$.

(iii) There exist no relations of the form

$$
\lambda_{i}=\sum_{j=1}^{m} n_{j} \cdot \lambda_{j}
$$

where $n_{j}(j=1, \cdots, m)$ are non-negative integers such that

$$
h>\sum_{j=1}^{m} n_{j}>1 \text {. }
$$

Conclusion: There exists a positive constant $h_{0}$, depending only on $\lambda_{i}(i=1, \cdots, m)$, with the following property: if $h>h_{0}$, there exist functions $u_{i}(x)(i=1, \cdots, m)$ of class $C^{1}$ satisfying (1.4), such that the system (1.1) is reduced to the form (1.2) by the substitution (1.3).

3. If (1.1) is transformed into (1.2) by (1.3), $u(x)$ must satisfy the system of partial differential equations 


$$
\sum_{i=1}^{m}\left(\sum_{j=1}^{m} a_{i j} x_{j}+f_{i}(x)\right) \cdot \partial_{i} u_{\nu}=\sum_{\mu=1}^{m} a_{\nu \mu} u_{\mu}-f_{\nu}(x) \quad(\nu=1, \cdots, m) .
$$

For we have, by operating $\frac{d}{d t}$ on both sides of (1.3),

$$
\frac{d y_{i}}{d t}=\frac{d x_{i}}{d t}+\sum_{\nu=1}^{m} \partial_{\nu} u_{i} \cdot \frac{d x_{\nu}}{d t} \quad(i=1, \cdots, m)
$$

from which (3.1) follows immediately by (1.1), (1.2) and (1.3). Conversely, if $u(x)$ is any function satisfying (3.1), then the substitution (1.3) will transform (1.1) into (1.2). Thus we have only to show the existence of $u(x)$ satisfying (1.4) and (3.1), if $h$ is sufficiently large.

\section{§ 3. Auxiliary Theorem.}

4. In this section we consider the system of semi-linear partial differential equations

$$
\sum_{i=1}^{m} P_{i}(x) \cdot \partial_{i} u_{\nu}=Q_{\nu}(x, u) \quad(\nu=1, \cdots, l)
$$

where $x=\left(x_{1}, \cdots, x_{m}\right)$ and $u=\left(u_{1}, \cdots, u_{l}\right)$ denote real vectors in $R^{m}$ and $R^{l}$ respectively. Let $P_{i}(x)$ be real-valued functions of class $C^{1}$ in an open domain $D \subset R^{m}$, such that

$$
\left(P_{1}(x), \cdots, P_{m}(x)\right) \neq(0, \cdots, 0) \quad(x \in D) .
$$

And $Q_{\nu}(x, u)$ be real-valued functions of class $C^{1}$ in

$$
\Omega=\left\{(x, u) \in R^{m+l}: x \in D,|u| \leqq \omega(x)\right\}
$$

where $\omega(x)$ is some positive-valued function of class $C^{1}$ in $D$. A curve $x=x(t)$ in $R^{m}$ is said to be a base characteristic of (4.1) if $x(t)$ satisfies the following system of ordinary differential equations:

$$
\frac{d x_{i}}{d t}=P_{i}(x) \quad(i=1, \cdots, m) .
$$

Let an $(m-1)$-dimensional manifold $M$ in $R^{m}$ be given by

$$
M: x_{i}=A_{i}\left(s_{1}, \cdots, s_{m-1}\right) \quad(i=1, \cdots, m)
$$

where $A_{i}(s)$ are functions of class $C^{1}$ in some domain $S \subset R^{m-1}$ such that $A(s)=\left(A_{1}(s), \cdots, A_{m}(s)\right) \in D$ for $s=\left(s_{1}, \cdots, s_{m-1}\right) \in S$. We assume that 


$$
\left|P_{i}(A(s)), \partial_{j} A_{i}(s) \quad \begin{array}{l}
i \downarrow 1, \cdots, m \\
j \rightarrow 1, \cdots, m-1
\end{array}\right|^{1)} \neq 0 \quad \text { for } \quad s \in S
$$

and that any base characteristic

$$
x=x(t, s),
$$

issuing from a point of $M$ so that $x(0, s)=A(s)$, exists on the interval: $0 \leqq t<\tau(s)$ where $\tau(s)$ is a continuous function on $S$, and that the set $X=\{x=x(t, s): 0 \leqq t<\tau(s), s \in S\}$ is filled up only onefold with all those curves $x=x(t, s)(s \in S)$, i.e. to any point $x \in X$ there corresponds just one $(t, s)$ such that $x=x(t, s), 0 \leqq t<\tau(s), s \in S$. Then we have easily

$$
\begin{aligned}
& \frac{\partial\left(x_{1}, \cdots, x_{m}\right)}{\partial\left(t, s_{1}, \cdots, s_{m-1}\right)} \\
& =\left|P_{i}(A(s)), \partial_{j} A_{i}(s) \quad \begin{array}{l}
i \downarrow 1, \cdots, m \\
j \rightarrow 1, \cdots, m-1
\end{array}\right| \cdot \exp \left(\int_{0}^{t} \sum_{i=1}^{m} \partial_{i} P_{i}(x)_{x=x(t) s)} d t\right) \neq 0,
\end{aligned}
$$

which shows that the 1-1 mapping (4.6) from $\{(t, s): s \in S, 0 \leqq t<\tau(s)\}$ onto $X$ and its inverse are both of class $C^{1}$.

By (4.6) the system (4.1) is reduced to the following system of ordinary differential equations, $s$ being a parameter:

$$
\frac{d u_{\nu}}{d t}=Q_{\nu}(x(t, s), u) \quad(\nu=1, \cdots, l) .
$$

We have then

$$
\partial_{t} \omega(x(t, s))=\left[\sum_{i=1}^{m} P_{i}(x) \cdot \partial_{i} \omega(x)\right]_{x=x(t, s)}
$$

and

$$
\partial_{t}|u(t, s)| \cdot|u(t, s)|=\sum_{\nu=1}^{t} u_{\nu}(t, s) \cdot Q_{\nu}(x(t, s), u(t, s))
$$

for any solution $u(t, s)$ of (4.7). Hence, we obtain easily the following auxiliary theorem which is our principal tool.

Auxiliary theorem. Under the conditions mentioned above, let the inequality

$$
\sum_{i=1}^{m} P_{i}(x) \partial_{i} \omega(x) \geqq \frac{1}{\omega(x)} \sum_{\nu=1}^{m} Q_{\nu}(x, u) \cdot u_{\nu}
$$

hold for any $x \in X$ such that $|u|=\omega(x)$. Then, for any function $B(s)=\left(B_{1}(s), \cdots, B_{l}(s)\right)$ of class $C^{1}$ on $S$ such that

1) an $(m, m)$-determinant. 


$$
|B(s)| \leqq \omega(A(s)),
$$

there exists a unique solution $u(x)$ of $(4.1)$ on $X$, such that

$$
u(A(s))=B(s)
$$

and

$$
|u(x)| \leqq \omega(x)
$$

for $x \in X$.

\section{$\S 4$. Estimation of $\boldsymbol{u}(\boldsymbol{x})$.}

5. Consider the system of partial differential equations

$$
\sum_{i=1}^{m}\left(\sum_{j=1}^{m} a_{i j} x_{j}+f_{i}(x)\right) \partial_{i} u_{\nu}=\sum_{\mu=1}^{m} a_{\nu \mu} \cdot u_{\mu}+g_{\nu}(x) \quad(\nu=1, \cdots, m)
$$

for which we have the following lemma.

Lemma. Let $A=\left(a_{i j}\right)$ and $f(x)$ satisfy the assumptions (i), (ii) and (iii) in the theorem. Let $g_{\nu}(x)(\nu=1, \cdots, m)$ be real-valued functions of class $C^{1}$ on some neighborhood of 0 , such that

$$
\left\{\begin{array}{l}
g(0)=0 \\
\left|\partial_{x} g(x)\right| \leqq G|x|^{p}
\end{array}\right.
$$

where $G$ and $p$ are positive constants.

Then there exists a constant $h_{0}>0$, which depends only on $\lambda_{i}(i=1, \cdots, m)$, with the following property: if $p>h_{0}$, the system (5.1) has a unique solution $u(x)$ in a neighborhood of 0 , such that

$$
u(x)=0 \text { on the cone } \sum_{i=1}^{k} x_{i}^{2}=\sum_{i=k+1}^{m} x_{i}^{2}{ }^{2}
$$

and

$$
\left|\partial_{x} u(x)\right| \leqq C \cdot G|x|^{p}
$$

where $C$ is a positive constant depending only on $\lambda_{i}(i=1, \cdots, m)$ and $p$.

6. Proof. By setting $P_{i}(x)=\sum_{j=1}^{m} a_{i j} x_{i}+f_{i}(x)$ and $Q_{\nu}(x, u)=\sum_{\mu=1}^{m} a_{\nu \mu} u_{\mu}$ $+g_{\nu}(x)$, the system (5.1) has the form (4.1). Without loss of generality we assume that $A=\left(a_{i j}\right)$ has the following form :

$$
\begin{array}{ll}
a_{i i}=\Re\left(\lambda_{i}\right) & (i=1, \cdots, m), \\
\Re\left(\lambda_{i}\right)>0 & \text { for } i \leqq k, \\
\Re\left(\lambda_{i}\right)<0 & \text { for } i>k .
\end{array}
$$

2) Cf. (i) $k=0$ means $\Re\left(\lambda_{i}\right)<0$ for all $i$, and $k=m$ means $\Re\left(\lambda_{i}\right)>0$ for all $i$. 
(ii)

$$
\begin{array}{ll}
a_{i j}=0 & \text { for } i \leqq k \text { and } j>k, \\
a_{i j}=0 & \text { for } i>k \text { and } j \leqq k
\end{array}
$$

(iii)

$$
\left|\sum_{i \neq j} a_{i j} x_{i} x_{j}\right| \leqq \delta|x|^{2}
$$

where $\delta$ is any prescribed positive number.

In what follows, we write $\sum_{\alpha}=\sum_{\alpha=1}^{k}$ and $\sum_{\beta}=\sum_{\beta=k+1}^{m}$. We suppose that $f$ and $g$ are functions of class $C^{1}$ on $U=\left\{x: \sum_{\alpha} x_{a}^{2} \leqq r^{2}, \sum_{\beta} x_{\beta}^{2} \leqq r^{2}\right\}$ where $r$ is a positive constant. We consider the case $0<k<m$. Because, if $k=0$ or $k=m$, the proof of the lemma will be simpler.

With sufficiently small $\varepsilon>0$ we set $^{3)}$

$$
S_{\varepsilon}(x)=\left\{\begin{array}{l}
\sum_{\alpha} x_{\alpha}^{2}-\sum_{\beta} x_{\beta}^{2} \quad \text { when } \quad \sum_{\alpha} x_{\alpha}^{2} \geqq \varepsilon^{2} \\
\sum_{\alpha} x_{\alpha}^{2}-\sum_{\beta} x_{\beta}^{2}-\frac{1}{2 \varepsilon^{2}}\left(\varepsilon^{2}-\sum_{\alpha} x_{\alpha}^{2}\right)^{2} \quad \text { when } \sum_{\alpha} x_{\alpha}^{2}<\varepsilon^{2},
\end{array}\right.
$$

and define a bounded region $U_{\varepsilon}$ by $U_{\varepsilon}=\left\{x \in U: S_{\varepsilon}(x) \geqslant 0\right\}$.

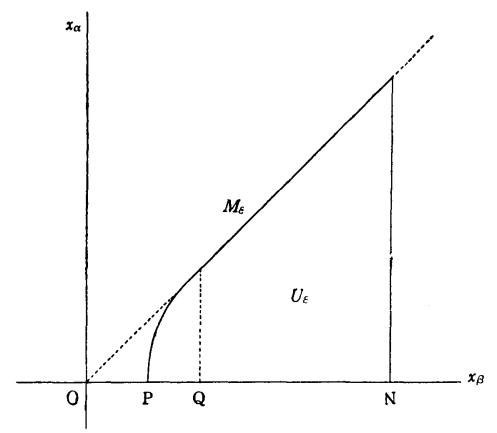

$$
\begin{aligned}
& \mathrm{N}=(r, 0) \\
& \mathrm{P}=\left(\frac{\sqrt{6}-\sqrt{2}}{2} \varepsilon, 0\right) \\
& \mathrm{Q}=(\varepsilon, 0)
\end{aligned}
$$

First we consider the solution of (5.1) in $U_{\varepsilon}$ vanishing on the $(m-1)$-dimensional manifold $M_{\varepsilon}=\left\{x \in U: S_{\varepsilon}(x)=0\right\}$. For the base characteristic $x=x(t)$ of $(5.1)$, we have

$$
\begin{aligned}
& \frac{1}{2} \cdot \frac{d}{d t} S_{\varepsilon}(x(t))=\sum_{\alpha} x_{a} \cdot \dot{x}_{a}-\sum_{\beta} x_{\beta} \cdot \dot{x}_{\beta} \\
& =\sum_{\alpha}\left(\sum_{i=1}^{m} a_{\alpha i} x_{i}+f_{a}(x)\right) x_{a}-\sum_{\beta}\left(\sum_{i=1}^{m} a_{\beta i} x_{i}+f_{\beta}(x)\right) x_{\beta} \\
& =\left(\sum_{\alpha} \sum_{i=1}^{m} a_{\omega i} x_{a s} x_{i}-\sum_{\beta} \sum_{i=1}^{m} a_{\beta i} x_{\beta} x_{i}\right)+\left(\sum_{\alpha} f_{a b}(x) x_{a b}-\sum_{\beta} f_{\beta}(x) x_{\beta}\right)>0
\end{aligned}
$$

3) For the case $k=0$ or $k=m$, we have to set $S_{\varepsilon}(x)=\sum_{i=1}^{m} x_{i}^{2}$. 
when $\varepsilon^{2}<\sum_{\alpha} x_{\alpha}^{2} \leqq r^{2}$, by taking $r$ small enough, and also when $\sum_{\alpha} x_{\alpha}^{2} \leqq \varepsilon^{2}$

$$
\frac{1}{2} \cdot \frac{d}{d t} S_{\varepsilon}(x(t))=\left\{1+\frac{1}{\varepsilon^{2}}\left(\varepsilon^{2}-\sum_{\alpha} x_{\alpha}^{2}\right)\right\} \sum_{\alpha} x_{\alpha} \cdot \dot{x}_{\alpha}-\sum_{\beta} x_{\beta} \cdot \dot{x}_{\beta}>0 .
$$

From these inequalities we see that, if $r>0$ is taken small enough, every base characteristic of (5.1) meeting $M_{\varepsilon}$ is transverse to $M_{\varepsilon}$, and that (4.5) will hold for this case with $M=M_{\varepsilon}$. In addition, since we have

$$
\frac{1}{2} \frac{d}{d t} \sum_{\alpha}\left(x_{\alpha}(t)\right)^{2}=\sum_{\alpha}\left(\sum_{i} a_{\omega i} x_{i}+f_{a b}(x)\right) x_{\alpha i}>0
$$

for any base characteristic $x(t)$, when $r$ is small enough, we see that $U_{\varepsilon}$ is filled up only onefold with the base characteristics issuing from $M_{\varepsilon}$. Therefore, we apply the auxiliary theorem to this case, setting $U_{\varepsilon}=X$. We set

$$
\varphi(x)=(1+\gamma) \sum_{\alpha} x_{\alpha}^{2}-\sum_{\beta} x_{\beta}^{2}
$$

and

$$
\omega(x)=W \cdot G \cdot \varphi(x)^{\frac{p+1}{2}}
$$

where $\gamma>0$ and $W>0$ will be determined later. Then

$$
\frac{\gamma}{2}|x|^{2} \leqq \varphi(x) \leqq(1+\gamma)|x|^{2}
$$

for every $x \in U_{\varepsilon}$. Thus we obtain

$$
\begin{aligned}
& \sum_{i=1}^{m} P_{i}(x) \partial_{i} \omega(x) \\
& =(p+1) \cdot W \cdot G \cdot \varphi(x)^{\frac{p-1}{2}}\left\{(1+\gamma) \sum_{\alpha} \sum_{j} a_{a j} x_{o s} x_{j}-\sum_{\beta} \sum_{j} a_{\beta j} x_{\beta} x_{j}\right. \\
& \left.+(1+\gamma) \sum_{\alpha} x_{a} f_{\alpha}(x)-\sum_{\beta} x_{\beta} f_{\beta}(x)\right\}
\end{aligned}
$$

and

$$
\sum_{\nu=1}^{m} Q_{\nu}(x, u) \cdot u_{\nu}=\sum_{\nu=1}^{m} \sum_{\mu=1}^{m} a_{\nu \mu} u_{\nu} u_{\mu}-\sum_{\nu=1}^{m} g_{\nu}(x) u_{\nu} .
$$

Now we set

$$
\Lambda_{0}=\min _{1 \leqq i \leqq m}\left|\Re\left(\lambda_{i}\right)\right|, \quad \Lambda_{1}=\max _{1 \leqq i \leqq m}\left|\Re\left(\lambda_{i}\right)\right| .
$$

Then we have

$$
\sum_{i=1}^{m} P_{i}(x) \cdot \partial_{i} \omega(x)>(p+1)\left(\Lambda_{0}-2 \delta\right) W \cdot G|x|^{2} \phi(x)^{\frac{p-1}{2}}
$$


and

$$
\sum_{\nu=1}^{m} Q_{\nu}(x, u) \cdot u_{\nu}<\left(\Lambda_{1}+\delta\right)|u|^{2}+|g(x)| \cdot|u|
$$

on $U_{\varepsilon}$ where $\delta$ is given by (6.1), taking $r$ small enough. From (6.8) it follows that, if $|u|=\omega(x)$ for some $x \in U_{\varepsilon}$, then

$$
\frac{1}{\omega(x)} \sum_{\nu=1}^{m} Q_{\nu}(x, u) u_{\nu}<\left(\Lambda_{1}+\delta\right) \omega(x)+G|x|^{p+1}
$$

and so

$$
\frac{1}{\omega(x)} \sum_{\nu=1}^{m} Q_{\nu}(x, u) u_{\nu}<W \cdot G\left(\Lambda_{1}+\delta\right)\left\{1+\gamma+\frac{\left(\frac{2}{\gamma}\right)^{\frac{p-1}{2}}}{\Lambda_{1} \cdot W}\right\}|x|^{2} \mathcal{P}(x)^{\frac{p-1}{2}}
$$

by virtue of (6.4) and (6.5). Thus, if we assume

$$
(p+1) \Lambda_{0}>\Lambda_{1},
$$

we have

$$
\sum_{i=1}^{m} P_{i}(x) \partial_{i} \omega(x)>\frac{1}{\omega(x)} \sum_{\nu=1}^{m} Q_{\nu}(x, u) \cdot u_{\nu}
$$

for any $x \in U_{\varepsilon}$ such that $|u|=\omega(x)$, from (6.7) and (6.9), by taking $\delta>0$ and $\gamma>0$ small enough and then $W$ large enough.

Let us assume hereafter that (6.10) holds. Then it follows from the auxiliary theorem that, when $r$ is small enough, there exists a unique solution $u(x ; \varepsilon)$ of $(5.1)$ on $U_{\varepsilon}$ which vanishes on $M_{\varepsilon}$, and that it satisfies

$$
|u(x ; \varepsilon)| \leqq \omega(x)=W \cdot G \cdot \varphi(x)^{\frac{p+1}{2}} \leqq G \cdot K \cdot|x|^{p+1} \quad \text { for } \quad x \in U_{\varepsilon}
$$

where $K=W(1+\gamma)^{\frac{p+1}{2}}$. Notice that $W$ and $r$ are taken independent of $\varepsilon>0$ in the above arguement.

\section{$\S 5$. Continuation of the Proof of the Lemma. Estimation of $\left|\partial_{x} u(x)\right|$.}

7. Next, let us prove that the inequality (5.4) holds for $u=u(x ; \varepsilon)$ on $U_{\varepsilon}$ with some constant $C>0$ independent of $\varepsilon$. In this paragraph we fix $\varepsilon>0$ and write $u_{\nu}(x)$ in place of $u_{\nu}(x ; \varepsilon)$ for simplicity.

In order to estimate $\left|\partial_{x} u(x)\right|$ on the manifold $M_{\varepsilon}$, we reduce the system (5.1) into

$$
\frac{d u_{\nu}}{d t}=\sum_{\mu=1}^{m} a_{\nu \mu} u_{\mu}+g_{\nu}(x(t, s)) \quad(\nu=1, \cdots, m)
$$


by the change of variables given by (4.6). Thus, if we set $u_{\nu}(t, s)$ $=u_{\nu}(x(t, s)), u=u(t, s)$ is the solution of (7.1) with the initial condition

$$
u(0, s) \equiv 0 \text {. }
$$

Therefore,

$$
\left\{\begin{array}{l}
\partial_{t} u(0, s) \equiv g(x(0, s)) \\
\partial_{s} u(0, s) \equiv 0
\end{array}\right.
$$

on $M_{\varepsilon}$. Let $\partial_{n} u(s)$ denote the normal derivative of $u(t, s)$ at $x=x(0, s)$ on $M_{\varepsilon}$, then we have

$$
\left[\frac{\partial_{t} u(t, s)}{\left\{\sum_{i=1}^{m}\left(\partial_{t} x_{i}(t, s)\right)^{2}\right\}^{\frac{1}{2}}}\right]_{t=0}=\partial_{n} u(s) \cdot \cos \theta(s)
$$

where $\theta(s)$ represents the angle between the base characteristic $x=x(t, s)$ and the normal of $M_{\varepsilon}$ at $x(0, s)$, i.e.

$$
\cos \theta(s)=\frac{(1+\sigma(x)) \cdot \sum_{\alpha} x_{\alpha} \cdot \partial_{t} x_{\alpha}-\sum_{\beta} x_{\beta} \cdot \partial_{t} x_{\beta}}{\left\{\sum_{i=1}^{m}\left(\partial_{t} x_{i}\right)^{2}\right\}^{\frac{1}{2}}\left\{(1+\sigma(x))^{2} \sum_{\alpha} x_{\alpha}^{2}+\sum_{\beta} x_{\beta}^{2}\right\}^{\frac{1}{2}}}
$$

where

Since

$$
\sigma(x)= \begin{cases}0 & \text { when } \sum_{\alpha} x_{\alpha}^{2} \geqq \varepsilon^{2} \\ \frac{1}{\varepsilon^{2}}\left(\varepsilon^{2}-\sum_{\alpha} x_{\alpha}^{2}\right) & \text { when } \sum_{\alpha} x_{\alpha}^{2}<\varepsilon^{2} .\end{cases}
$$

(7. 6) $\quad(1+\sigma(x)) \cdot \sum_{\alpha} x_{\omega} \cdot \partial_{t} x_{\alpha}-\sum_{\beta} x_{\beta} \cdot \partial_{t} x_{\beta}>\frac{1}{2} \Lambda_{0}|x|^{2} \quad\left(x \in U_{\mathrm{z}}\right)$

and

$$
(1+\sigma(x))^{2} \cdot \sum_{\alpha} x_{\alpha}^{2}+\sum_{\beta} x_{\beta}^{2}<2|x|^{2},
$$

when $r$ is sufficiently small, we obtain, from (7.3), (7.4), (7.5), (7.6) and (7.7),

Hence

$$
\left|\partial_{n} u\right|<\frac{2 \sqrt{2}}{\Lambda_{0}} \cdot \frac{|g(x)|}{|x|} .
$$

$$
\left|\partial_{n} u\right|<K^{\prime} \cdot G|x|^{p}
$$

on $M_{\varepsilon}$ with some constant $K^{\prime}>0$ independent of $\varepsilon$. Thus, from (7.3) and (7.8), we see

$$
\left|\partial_{x} u(x ; \varepsilon)\right| \leqq K^{\prime} \cdot G|x|^{p} \quad\left(x \in M_{\varepsilon}\right) .
$$


Now, operating $\partial_{\mu}$ on both sides of (5.1) and setting $\partial_{\mu} u_{\nu}=u^{\nu \mu}$, we have

$$
\begin{gathered}
\sum_{i=1}^{m}\left(\sum_{j=1}^{m} a_{i j} x_{j}+f_{i}(x)\right) \partial_{i} u^{\nu \mu} \\
=\sum_{j=1}^{m} a_{\nu j} u^{j \mu}-\sum_{i=1}^{m}\left(a_{i \mu}+\partial_{\mu} f_{i}(x)\right) u^{\nu i}+\partial_{\mu} g_{\nu}(x) \quad(\nu, \mu=1, \cdots, m)
\end{gathered}
$$

which has also the form (4.1) with unknown functions $u^{\nu \mu}$. Let us assume first that $f$ and $g$ are functions of class $C^{2}$ in $U$ and apply the auxiliary theorem to (7.10). We set

$$
\begin{gathered}
u^{\prime}=\left(u^{1,1}, u^{1,2}, \cdots, u^{m, m}\right) \quad\left(\in R^{m 2}\right) \\
P_{i}(x)=\sum_{j=1}^{m} a_{i j} x_{j}+f_{i}(x) \\
Q^{\nu \mu}\left(x, u^{\prime}\right)=\sum_{j=1}^{m} a_{\nu j} u^{j \mu}-\sum_{i=1}^{m}\left(a_{i \mu}+\partial_{\mu} f_{i}(x)\right) \cdot u^{\nu i}+\partial_{\mu} g_{\nu}(x) \quad(\nu, \mu=1, \cdots, m) \\
\text { and } \quad \omega^{\prime}(x)=W^{\prime} \cdot G \cdot \phi^{\prime}(x)^{\frac{p}{2}} \quad \text { where } \quad \phi^{\prime}(x)=\left(1+\gamma^{\prime}\right) \sum_{\alpha} x_{\alpha}^{2}-\sum_{\beta} x_{\beta}^{2} .
\end{gathered}
$$

Then, if we assume

$$
p \Lambda_{0}>\tilde{\Lambda} \equiv \max _{i, j}\left|\Re\left(\lambda_{i}\right)-\mathfrak{R}\left(\lambda_{j}\right)\right|,
$$

and if we take $r$ small enough, we have

$$
\sum_{i=1}^{m} P_{i}(x) \partial_{i} \omega^{\prime}(x)>\frac{1}{\omega^{\prime}(x)} \sum_{=1}^{m} \sum_{\mu=1}^{m} Q^{\nu \mu}\left(x, u^{\prime}\right) \cdot u^{\nu \mu}
$$

for $x$ such that $\omega^{\prime}(x)=\left|u^{\prime}\right|$ in $U_{\varepsilon}$, taking $W^{\prime}$ and $\gamma^{\prime}>0$ appropriately. By the auxiliary theorem and (7.9) we thus get

$$
\left|\partial_{x} u(x ; \varepsilon)\right| \leqq C \cdot G|x|^{p}
$$

in $\dot{U}_{\varepsilon}$ where $C>0$ is a constant independent of $\varepsilon$. In the above consideration we can also choose $r$ independent of $\varepsilon$. Let us write

$$
h_{0}=\max \left(\frac{\Lambda_{1}}{\Lambda_{0}}-1, \tilde{\Lambda}\right)
$$

and assume $p>h_{0}$ hereafter, from which (6.10) and (7.11) follow.

We will study in 9 . as to the case that $f$ and $g$ are functions of class $C^{1}$.

8. Notice that $C$ of $(7.12)$ and $r$ can be chosen independent of $\varepsilon>0$ which is sufficiently small. Now we consider $\varepsilon$ as a variable tending to zero. We see easily $U_{\varepsilon} \subset U_{\varepsilon}^{\prime}$ as $\varepsilon>\varepsilon^{\prime}>0$, and $v=u\left(x ; \varepsilon^{\prime}\right)-u(x ; \varepsilon)$ must satisfy on $U_{\varepsilon}$

$$
\sum_{i=1}^{m}\left(\sum_{j=1}^{m} a_{i j} x_{j}+f_{i}(x)\right) \partial_{i} v_{\nu}=\sum_{\mu=1}^{m} a_{\nu \mu} v_{\mu} \quad(\nu=1, \cdots, m) .
$$


From (6.12) and (7.12) we see easily that

and

$$
|v| \leqq 2^{\frac{p+3}{2}} K \cdot G \cdot \varepsilon^{p+1}
$$

$$
\left|\partial_{x} v\right| \leqq 2^{\frac{p+2}{2}} C \cdot G \cdot \varepsilon^{p}
$$

hold for $v=u\left(x ; \varepsilon^{\prime}\right)-u(x ; \varepsilon)$ on $M_{\mathrm{\varepsilon}}$. Notice that $\min _{x \in U_{\varepsilon}}|x|=\frac{\sqrt{6}-\sqrt{2}}{2} \varepsilon$, and we have for any $q>0$

$$
\left\{\begin{aligned}
&|v| \leqq K_{0} \cdot \varepsilon^{p-q}|x|^{q+1} \\
&\left|\partial_{x} v\right| \leqq K_{1} \cdot \varepsilon^{p-q}|x|^{q}
\end{aligned}\right.
$$

on $M_{\varepsilon}$ where $K_{0}$ and $K_{1}$ are constants not depending on $\varepsilon$ and $\varepsilon^{\prime}$.

We now choose $q$ so that $p>q>h_{0}$. The system (8.1) is a special case of (5.1) with $g(x) \equiv 0$, and we get similarly as (6.12) and (7.12)

$$
\left\{\begin{aligned}
\left|u\left(x ; \varepsilon^{\prime}\right)-u(x ; \varepsilon)\right| & \leqq K^{\prime} \cdot \varepsilon^{p-q}|x|^{q+1} \\
\left|\partial_{x} u\left(x ; \varepsilon^{\prime}\right)-\partial_{x} u(x ; \varepsilon)\right| & \leqq K^{\prime} \cdot \varepsilon^{p-q}|x|^{q}
\end{aligned}\right.
$$

in $U_{\varepsilon}$ where $K^{\prime}$ is some positive constant independent of $\varepsilon$ and $\varepsilon^{\prime}$. Thus we see that, as $\varepsilon \rightarrow 0, u_{\nu}(x ; \varepsilon)$ and $\partial_{\mu} u_{\nu}(x ; \varepsilon)$ tend to certain functions $u_{\nu}(x)$ and their derivatives $\partial_{\mu} u_{\nu}(x)$ respectively. Clearly this $u(x)$ is a solution of (5.1), vanishing on the manifold : $\sum_{\alpha} x_{\alpha}^{2}=\sum_{\beta} x_{\beta}^{2}$ and satisfying (5.4) in $U_{1}=\left\{x: \sum_{\alpha} x_{\alpha}^{2} \leqq r^{2}, \sum_{\beta} x_{\beta}^{2} \leqq \sum_{\alpha} x_{\alpha}^{2}\right\}$.

Quite similarly as above, we can prove the existence of a solution $u(x)$ of (5.1) vanishing on $\sum_{\alpha} x_{\alpha}^{2}=\sum_{\beta} x_{\beta}^{2}$ and satisfying (5.4) in $U_{2}$ $=\left\{x: \sum_{\beta} x_{\beta}^{2} \leqq r^{2}, \sum_{\alpha} x_{\alpha}^{2} \leqq \sum_{\beta} x_{\beta}^{2}\right\}$.

9. Now it remains to prove our lemma when $f$ and $g$ are functions of class $C^{1}$. We construct approximation sequences $\left\{f^{n}(x)\right\}_{n=1}^{\infty}$ and $\left\{g^{n}(x)\right\}_{n=1}^{\infty}$ of vector functions of class $C^{2}$ such that ${ }^{4)}$

$$
\left\{\begin{array}{l}
f^{n}(0)=g^{n}(0)=0 \\
f^{n}(x)=p(x)+q^{n}(x)^{5} \\
\left|\partial_{x} f^{n}(x)-\partial_{x} f(x)\right| \leqq \frac{1}{n}|x|^{n} \\
\left|\partial_{x} g^{n}(x)-\partial_{x} g(x)\right| \leqq \frac{1}{n}|x|^{n} .
\end{array}\right.
$$

4) $f^{n}(x)$ and $g^{n}(x)$ have only to be of class $C^{2}$ in $U$ excepting $x=0$.

5) c. f. (2.1). 
Then there exists a system $u^{n}(x)=\left(u_{1}^{n}(x), \cdots, u_{m}^{n}(x)\right)$ of functions of class $C^{2}$ satisfying

$$
\sum_{i=1}^{m}\left(\sum_{j=1}^{m} a_{i j} x_{j}+f_{i}^{n}(x)\right) \partial_{i} u_{\nu}=\sum_{\mu=1}^{m} a_{\nu \mu} u_{\mu}+g_{\nu}^{n}(x) \quad(\nu=1, \cdots, m),
$$

such that

$$
\left\{\begin{aligned}
\left|u^{n}(x)\right| & \leqq C \cdot K_{2}|x|^{p+1} \\
\left|\partial_{x} u^{n}(x)\right| & \leqq C \cdot K_{3}|x|^{p}
\end{aligned}\right.
$$

in $U$ where $K_{2}$ and $K_{3}$ are constants not depending on $n$. For $u^{n}(x)-u^{n^{\prime}}(x)$ $\left(n \leqq n^{\prime}\right)$ we have

$$
\sum_{i=1}^{m}\left(\sum_{j=1}^{m} a_{i j} x_{j}+f_{i}^{n}(x)\right) \partial_{i}\left(u_{\nu}^{n}-u_{\nu}^{n^{\prime}}\right)=\sum_{\mu=1}^{m} a_{\nu \mu}\left(u_{\mu}^{n}-u_{\mu}^{n^{\prime}}\right)+h_{\nu}^{n, n^{\prime}}(x),
$$

where

$$
h_{\nu}^{n, n^{\prime}}(x)=\left\{g_{\nu}^{n}(x)-g_{\nu}^{n^{\prime}}(x)\right\}+\partial_{i} u_{\nu}^{n^{\prime}}\left\{f_{\nu}^{n^{\prime}}(x)-f_{\nu}^{n}(x)\right\}
$$

and so

$$
h^{n, n^{\prime}}(0)=0 \quad \text { and } \quad\left|\partial_{x} h^{n, n^{\prime}}(x)\right| \leqq \frac{H}{n}|x|^{p}
$$

with a constant $H>0$ not depending on $n$ and $n^{\prime}$. Therefore we see that, as $n \rightarrow \infty, u^{n}(x)$ tend to the desired solution of (5.1). The proof of the lemma is thus completed.

\section{§6. Proof of the Main Theorem.}

10. Let us now turn to the system (3.1),

$$
\sum_{i=1}^{m}\left(\sum_{j=1}^{m} a_{i j} x_{j}+f_{i}(x)\right) \partial_{i} u_{\nu}=\sum_{\mu=1}^{m} a_{\nu \mu} u_{\mu}-f_{\nu}(x) \quad(\nu=1, \cdots, m)
$$

where $f_{\nu}(x)=p_{\nu}(x)+q_{\nu}(x)$. First, let us consider

1) $\sum_{i=1}^{m}\left(\sum_{j=1}^{m} a_{i j} x_{j}+p_{i}(x)\right) \partial_{i} u_{\nu}=\sum_{\mu=1}^{m} a_{\imath \mu} u_{\mu}-p_{\nu}(x) \quad(\nu=1, \cdots, m)$.

If there exist no relations of the form $\lambda_{i}=\sum n_{j} \lambda_{j}$ where $n_{j}$ are non-negative integers such that $\sum_{j} n_{j}>1$, we can construct infinite series of the form

$$
\sum_{\substack{p_{i} \geqq 0 \\ p_{1}+\cdots+p_{m} \geqq 2}}^{c_{p_{1}}^{\nu}, \cdots, p_{m}} \cdot x_{1}^{p_{1}} \cdot \cdots \cdot x_{m}^{p_{m}}
$$

with real coefficients $c_{p_{1}, \cdots, p_{m}}^{\nu}$, such that $u_{\nu}=\sum c_{p_{1}, \cdots, p_{m}}^{\nu} \cdot x_{1}^{p_{1}} \cdots x_{m}^{p_{m}}$ $(\nu=1, \cdots, m)$ satisfy $(10.1)$ formally. To see this, make a change of variables, $x=T \cdot y$ and $u=T \cdot w$, by a (complex) matrix $T$ transforming $A=\left(a_{i j}\right)$ into Jordan's canonical form $T^{-1} \cdot A \cdot T$, and consider about the (complex) system thus obtained. 
Setting

$$
\dddot{u}_{\nu}(x)=\sum_{2 \leqq p_{1}+\cdots+p_{m} \leqq h} c_{p_{1} \cdots p_{m}}^{\nu} \cdot x_{1}^{p_{1}} \cdots x_{m}^{p_{m}} \quad(\nu=1, \cdots, m)
$$

and

$$
\grave{u}_{\nu}=u_{\nu}-u_{\nu}(x) \quad(\nu=1, \cdots, m),
$$

we reduce $(3.1)$ to the system

$$
\sum_{i=1}^{m}\left(\sum_{j=1}^{m} a_{i j} x_{j}+f_{i}(x)\right) \partial_{i} \grave{u}_{\nu}=\sum_{\mu=1}^{m} a_{\nu \mu} \grave{u}_{\mu}+\tilde{f}_{\nu}(x) \quad(\nu=1, \cdots, m)
$$

where

$$
\tilde{f}_{\nu}(x)=\sum_{\mu=1}^{m} a_{\nu \mu} \tilde{u}_{\mu}(x)-\sum_{i=1}^{m}\left(\sum_{j=1}^{m} a_{i j} x_{j}+f_{i}(x)\right) \partial_{i} \tilde{u}_{\nu}(x)-f_{\nu}(x)
$$

In order to define $u^{\prime}(x)$ as above, we have only to assume that condition (iii) in the theorem $(\S 2)$ is satisfied.

By (2.1) we have

$$
\tilde{f}_{\nu}(x)=\tilde{p}_{\nu}(x)-\tilde{q}_{\nu}(x)
$$

where

$$
\tilde{p}_{\nu}(x)=\sum_{\mu=1}^{m} a_{\nu \mu} \tilde{u}_{\mu}(x)-p_{\nu}(x)-\sum_{i=1}^{m}\left(\sum_{j=1}^{m} a_{i j} x_{j}+p_{i}(x)\right) \partial_{i} \dot{u}_{\nu}(x)
$$

and

$$
\tilde{q}_{\nu}(x)=q_{\nu}(x)+\sum_{i=1}^{m} q_{i}(x) \partial_{i} \dot{u}_{\nu}(x),
$$

and it follows from the definition of $u(x)$ that $\tilde{p}_{\nu}(x)$, polynomials in $x_{i}(i=1, \cdots, m)$, contain no terms of degree $\leqq h$. Therefore, by (2.2)

$$
\left\{\begin{array}{l}
\tilde{f}_{\nu}(0)=0 \quad(\nu=1, \cdots, m), \\
\left|\partial_{x} \tilde{f}(x)\right| \leqq \tilde{K} \cdot|x|^{h}
\end{array}\right.
$$

where $\tilde{K}$ is a positive constant. Using the lemma, we thus see that there exists a solution $\grave{u}(x)=\left(\grave{u}_{1}(x), \cdots, \grave{u}_{m}(x)\right)$ of $(10.4)$ in some neighborhood of $x=0$, such that

and

$$
\grave{u}_{i}(0)=0 \quad(i=1, \cdots, m)
$$

$$
\left|\partial_{x} \grave{u}(x)\right| \leqq C \cdot \tilde{K}|x|^{h}
$$

with some constant $C>0$. We set

$$
u_{\nu}(x)=u_{\nu}(x)+\grave{u}_{\nu}(x) \quad(\nu=1, \cdots, m)
$$

and obtain those functions $u_{\nu}(x)(\nu=1, \cdots, m)$ whose existence was claimed 
in the theorem. The proof of the theorem is thus completed.

(Received November 12, 1957)

\section{Literature}

[1] E. Picard: Traité d'analyse. III. Chap. 1.

[2] M. Urabe: On solutions of the linear homogeneous partial differential equation in the vicinity of the singularity. I, II. J. Sci. Hiroshima Univ. Ser. A. 14 (1950), 115-126, 195-207.

[3] C. L. Siegel: Ueber die Normalform analytischer Differentialgleichungen in der Nähe einer Gleichgewichtslösung. Nachr. Akad. Wiss. Göttingen. Math.Phys. Kl. (1952), 21-30. 